\title{
Mitral Valve Replacement with Thymectomy in a Patient with Ocular Myasthenia Gravis: Case Report
}

\author{
Ovidiu Stiru, MD, PhD, Mihai Stefan, MD, Roxana Carmen Geana, MD, Diana Sorostinean, MD, \\ Răzvan Radu, MD, PhD, Daniela Filipescu, MD, PhD, Ovidiu Chioncel, MD, PhD, \\ Vlad Anton Iliescu, MD, PhD
}

Emergency Institute for Cardiovascular Diseases “Prof. Dr. C. C. Iliescu,” Bucharest, Romania

\section{ABSTRACT}

Patients diagnosed with ocular myasthenia gravis (MG) and mitral valve disease represent a significant perioperative management problem, especially for the anaesthesiologist, due to complex inter-actions between the disease, drugs to treat the disease, and anaesthetic agents, such as neuromuscular blocking agents (NMBAs).

This paper describes the successful management of a 31-year-old female with mitral valve stenosis and ocular MG who was diagnosed with MG 4 years prior to the indication for cardiac surgery. Preoperatively, the patient was under treatment with Pyridostigmine and Prednisone. Mitral valve replacement and full thymectomy were performed, under general anaesthesia, using Fentanyl, Sevoflurane and low doses of non-depolarising NMBAs.

The postoperative course was uneventful, the patient was extubated at 6 hours postoperatively, in-tensive care unit stay was 48 hours, and the patient was discharged after 6 days without any compli-cations. After 3 months, at the follow-up examination, the patient's ocular symptoms (eyelid ptosis) disappeared.

\section{INTRODUCTION}

MG is a disease characterized by muscle weakness and fatigue that worsens with exercise and im-proves with rest. It is an autoimmune disorder, mainly caused by the production of antibodies against the acetylcholine receptor in the skeletal muscles, resulting the failure of neuromuscular transmission [Vincent 2011]. It was first described in 1672 by physician Thomas Willis [Drachman 1994]. Although MG extensively has been studied [Conti-Fine 2006], there are few reported cases that associate mitral valve disease [Narin 2009; Aps 1987; Wang 2011].

The annual incidence of MG has been estimated between 0.25 and 2 per 100,000 inhabitants [Vincent 2011], but can be higher in selected populations [Kalb 2002]. It has two peaks,

Received fune 23, 2019; accepted fuly 24, 2019

Correspondence: Ovidiu Stiru, MD, PhD, Emergency Institute for Cardiovascular Diseases "Prof. Dr. C. C. Iliescu" Sos. Fundeni 258, sector 2, 022328 Bucharest, Romania; Phone/Fax: +400722207286 (e-mail: ovidiu_ stiru@yahoo.com). the first one between the second and third decades of life, mainly affecting women, and the second, between the sixth and seventh decades [Drachman 1994].

Ocular MG occurs in $15 \%$ of cases and the muscles involved are only the extraocular, levator palpebrae superioris and orbicularis oculi, while pupillary muscles are spared; in most patients with ocular MG the symptoms vary from diplopia to blurred vision and some have ptosis of the eyelids [Vaphiades 2012]. In generalized MG, the muscles of the neck and diaphragm are affected, and this form occurs in approximately $85 \%$ of cases [Drachman 1994].

Mechanical ventilation is mandatory during a myasthenic crisis, in order for the patient to survive [Blichfeldt-Lauridsen 2012]. In 50\% of cases of ocular MG, antibodies against acetylcholine recep-tors (AchR) are found [Drachman 1994], and up to half of these patients have antibodies against muscle-specific tyrosine kinase (MuSK) [Conti-Fine 2006].

While still debated, thymectomy is a feasible and effective treatment, both in thymomatous and nonthymomatous MG cases [Aydin 2017]. Careful intraoperative and postoperative management, with general anaesthesia and appropriate use of NMBAs, as well as meticulous assessment of the patient's neurologic and cardiac status are needed in order to avoid life-threatening complications [Blichfeldt-Lauridsen 2012; Ishimura 1998].

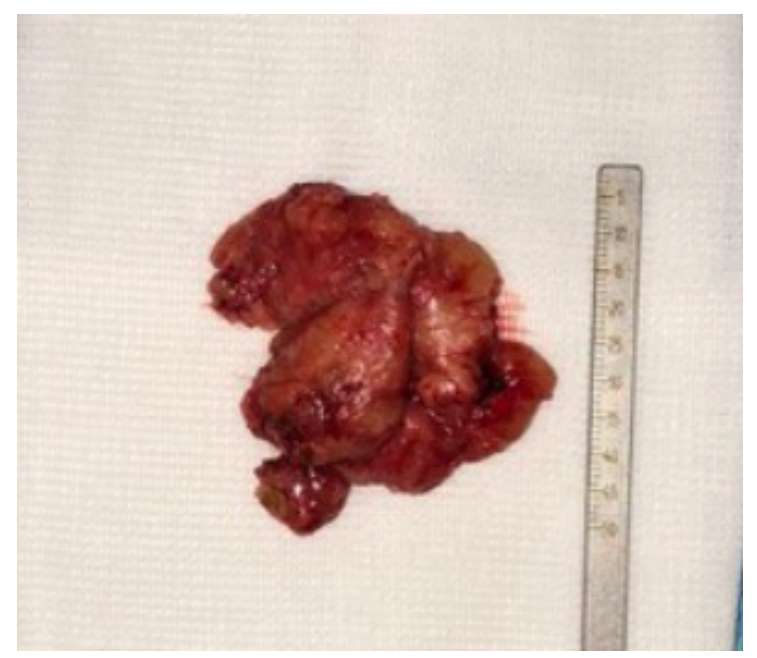

Figure 1. Excised thymus. 


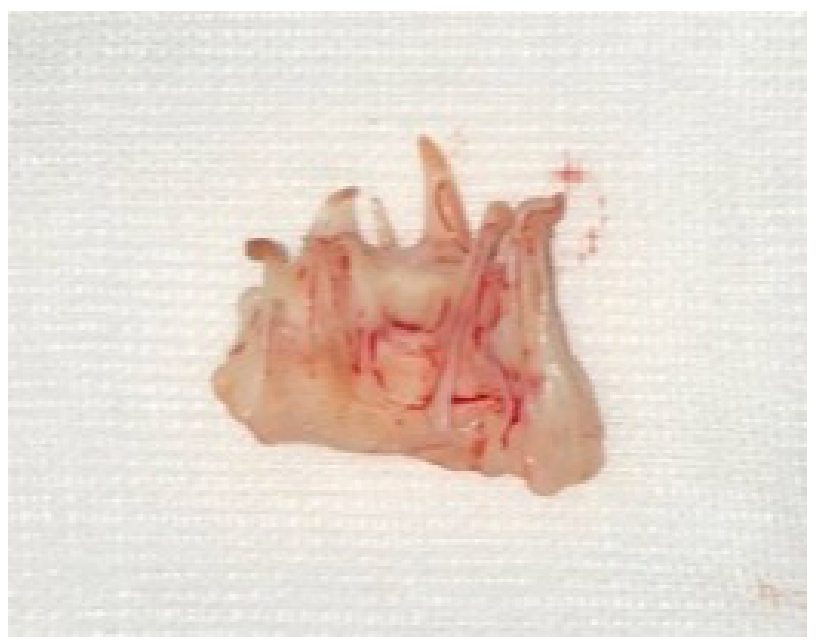

Figure 2. Excised mitral valve.

In this paper, we present a successful mitral valve replacement and thymectomy in a patient with ocular MG, using normothermic cardiopulmonary bypass (CPB).

\section{CASE PRESENTATION}

We report the case of a 31-year-old female patient presented in our center with severe mitral steno-sis who had a history of mild chest pain, exertional dyspnoea, and fatigue for over 1 year.

The patient had been diagnosed with ocular MG 4 years prior to admission. Neurologic examination showed some weakness of the bulbar and extra-ocular muscles, mainly occurring in the early morn-ing, with occasional ptosis of one or both palpebrae. Eye weakness often changed over the course of a day, aggravating in the evening and improving after resting for a few minutes. The patient did not have difficulty swallowing, speaking or breathing, nor did she experience weakness of the arms or legs.

Transthoracic echocardiography detected a severe mitral valve stenosis (pressure half-time $202 \mathrm{~ms}$, mean transvalvular gradient $11 \mathrm{mmHg}$ ), a left ventricular ejection fraction of $55 \%$, with no pulmo-nary hypertension. The coronary angiography found normal coronary arteries. ECG showed normal sinus rhythm, and haematological and biochemical tests also came back normal. Immunological tests did not detect AchR and MuSK antibodies. Chest X-ray findings included left atrial enlarge-ment. At the pre-anaesthetic examination, there was no diplopia or ptosis, swallowing was normal, and the patient did not have any other comorbidities. Daily oral drug therapy included Pyridostig-mine $60 \mathrm{mg}$ twice per day and Prednisone $10 \mathrm{mg}$ every other day.

The patient was scheduled for an elective mitral valve replacement surgery and thymectomy using a standard CPB. Preoperatively, the patient didn't receive any hypnotic pre-medication, but received her usual morning dose of Pyridostigmine and Prednisone. Intraoperative monitoring

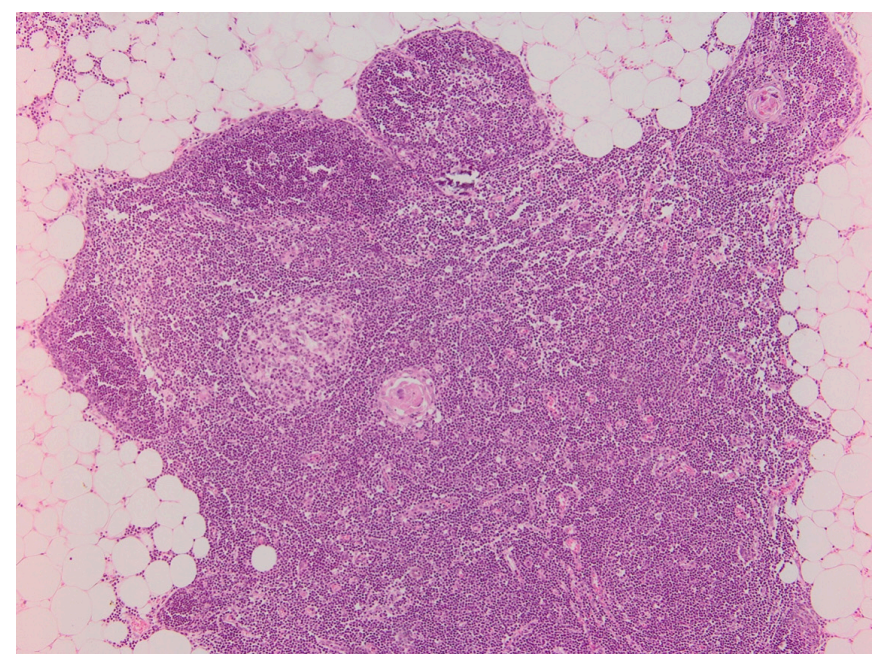

Figure 3. Hystological specimen - normal thymus gland (H\&E staining paraffin sections; magnifi-cation $\times 10$ ).

included continuous electrocardiography (ECG), peripheral pulse oximetry, diuresis, body temperature measuring at the bladder, capnography, train-of-four (TOF), invasive blood pressure via a left radial cathe-ter, and central venous pressure monitoring via a triple lumen central venous line.

General anaesthesia was induced using midazolam $3.5 \mathrm{mg}$, fentanyl $0.3 \mu \mathrm{g}$ and rocuronium $20 \mathrm{mg}$, with uneventful tracheal intubation, under direct laryngoscopy. Anaesthesia was maintained with sevoflurane and analgesia provided with fentanyl, $3 \mu \mathrm{g} / \mathrm{kg} / \mathrm{hour}$, with supplemental boluses at the anaesthesiologist's clinical judgement (total dose - $1.2 \mathrm{mg}$ ).

Surgery was performed using median sternotomy. At inspection of the retrosternal space, we found the $5 \mathrm{~cm} / 4 \mathrm{~cm}$ thymus mass which was not invasive (Figure 1) and a total thymectomy was per-formed before opening the pericardium. We performed standard cannulation of the ascending aorta and double, superior and inferior vena cava. Normothermic CPB was established and aortic crossclamping was done. Cold blood anterograde cardioplegia was used, a left atriotomy was carried out, the mitral valve was totally excised (Figure 2), and a bi-leaflet mechanical valve prosthesis (Car-bomedics no. 29) was implanted without any perioperative complication. Total CPB time was 58 minutes, with an uneventful weaning.

The patient was extubated 6 hours postoperatively, using TOF monitoring for ensuring adequate NMBA effect disappearance (TOF ratio $>0.9$ ) and did not require pharmacologic reversal. There was no evidence of mediastinal bleeding, and the chest tubes were removed on the second postoperative day. MG therapy was reprised from the first postoperative day. Intensive care unit stay was 48 hours, and the patient was discharged from the hospital after 6 days. The histopathological examination of both surgical specimens revealed a normal thymus gland (Figure 3 ) and rheumatic valvular mitral disease. Follow-up examination at 3 months showed a dose reduction by half in MG treatment and disappearance of the palpebral ptosis. 


\section{DISCUSSION}

MG is an autoimmune disease with serious potential respiratory complications due to the decrease in the number of active AChR at the neuromuscular junction [Conti-Fine 2006]. Its combination with cardiac disease is rare [Shivamurthy 2014], and anaesthesia required for cardiac surgery may cause life-threatening complications [Ishimura 1998].

MG patients with thymoma present have a higher prevalence of cardiac manifestations, and thymectomy has been reported to significantly improve the clinical condition [Blichfeldt-Lauridsen 2012]. Since heart failure symptoms may result from both MG and mitral valve disease (MVD), with substantial overlap between two pathologies, the coexistence of MVD is not clinically apparent and may be detected later in the course of the disease. Fortunately in our patient, dyspnoea was not a consequence of respiratory muscle weakness.

Meticulous assessment of the patient's neurologic and cardiac status were needed in order to avoid life-threatening complications. Treatment plans for patients with MG are based on the severity of the disease and include anticholinesterases, corticosteroids, immunosuppressants, plasmapheresis, immunoglobulin, and thymectomy therapies [Vincent 2011]. Assuming thymus would be the site of antibody formation, thymectomy is considered a viable treatment in nonthymomatous patients, which represent $85 \%$ to $90 \%$ of total [Aydin 2017], and studies show that it can add clinical benefit even in seronegative patients (up to $20 \%$ of patients) [Yuan 2007]. This has been the case with our patient, which has shown clinical benefit (dose reduction at clinical follow-up) after thymectomy.

In literature, there have been very few reports describing perioperative anaesthetic management in MG patients undergoing cardiac surgery [Wang 2011; Ishimura 1998].

In MG patients, anaesthetic doses vary individually, patients have an unpredictable response to muscle relaxants and an increased susceptibility to postoperative respiratory failure, resulting in prolonged dependence on mechanical ventilation, especially after myasthenic crisis [Li 2014]. Ro-curonium, cisatracurium, and vecuronium all are relatively short-acting nondepolarizing neuromus-cular blocking agents, and they have a similar effect on MG patients [Blichfeldt-Lauridsen 2012]. Muscle relaxant monitoring is mandatory [Dillon 2014], and pharmacologic reversal of NMBAs using suggamadex needs to be an option when using rocuronium or vecuronium.

\section{CONCLUSION}

We consider successful management of simultaneous mitral valve replacement and thymectomy in patients with MG can be achieved by methodical preoperative evaluation and careful perioperative management, thus improving long-term outcome.

\section{REFERENCES}

Aps C, O'Sullivan G. 1987. Myasthenia gravis and cardiac surgery. Br J Anaesth. Feb;59(2):265-8.

Wang Q, Xu Z, Lin B, Lu F, Cui Y. 2011. Simultaneous Operation for Multiple Valvular Disease and Myasthenia in a Woman. Heart Lung Circ. Feb; 20(2):130-1.

Aydin Y, Ulas AB, Mutlu V, Colak A, Eroglu A. 2017. Thymectomy in Myasthenia Gravis. Eurasian J Med. Mar 31;49(1):48-52.

Blichfeldt-Lauridsen L, Hansen BD. 2012. Anesthesia and myasthenia gravis. Acta Anaesthesiol Scand. Jan;56(1):17-22.

Conti-Fine BM, Milani M, Kaminski HJ. 2006. Myasthenia gravis: past, present, and future. J Clin Invest. Nov 1;116(11):2843-54.

Dillon F. 2014. Anesthesia Issues in the Perioperative Management of Myasthenia Gravis. Semin Neurol. Jul 1;24(01):83-94.

Drachman D. 1994. Myasthenia Gravis. N Engl J Med. 330(25):1797-810.

Ishimura H, Sata T, Matsumoto T, Takizuka A, Shigematsu A. Anesthetic Management of a Patient with Myasthenia Gravis During Hypothermic Cardiopulmonary Bypass. 1998. J Clin Anesth. May;10(3):228-31.

Kalb B, Matell G, Pirskanen R, Lambe M. 2002. Epidemiology of Myasthenia gravis: A Population-Based Study in Stockholm, Sweden. Neuroepidemiology. 21(5):221-5.

Li K-K, Qian K, Feng Y-G, Guo W, Tan Q-Y, Deng B. 2017. Predictive factors of prolonged me-chanical ventilation, overall survival, and quality of life in patients with post-thymectomy myas-thenic crisis. World J Surg Oncol [Internet]. 2017 Dec [cited 2018 Aug 28];15(1).

Narin C, Sarkilar G, Tanyeli O, Ege E, Yeniterzi M. 2009. Successful mitral valve surgery in a patient with myasthenia gravis. J Card Surg. 24(2):210-2.

Shivamurthy P, Parker MW. 2014.Cardiac manifestations of myasthenia gravis: A systematic re-view. IJC Metab Endocr. Nov;5:3-6.

Vaphiades MS, Bhatti MT, Lesser RL. 2012. Ocular myasthenia gravis: Curr Opin Ophthalmol. Nov;23(6):537-42.

Vincent A, Palace J, Hilton-Jones D. 2001. Myasthenia gravis. Lancet. 357:2122-8.

Yuan HK, Huang B-S, Kung S-Y, Kao K-P. 2007. The effectiveness of thymectomy on seronega-tive generalized myasthenia gravis: comparing with seropositive cases. Acta Neurol Scand. Mar;115(3):181-4. 